\title{
Reginaldo Chayax Huex (1939-2020)
}

Scott Atran, Ximena Lois y Valentina Vapnarsky

\section{OpenEdition}

Journals

Edición electrónica

URL: https://journals.openedition.org/jsa/18886

DOI: 10.4000/jsa.18886

ISSN: 1957-7842

Traducción(es):

Reginaldo Chayax Huex (1939-2020) - URL : https://journals.openedition.org/jsa/19243 [en]

\section{Editor}

Société des américanistes

\section{Edición impresa}

Fecha de publicación: 15 diciembre 2020

Paginación: 269-273

ISSN: 0037-9174

\section{Referencia electrónica}

Scott Atran, Ximena Lois y Valentina Vapnarsky, «Reginaldo Chayax Huex (1939-2020)», Journal de la Société des américanistes [En línea], 106-2 | 2020, Publicado el 30 diciembre 2020, consultado el 16 septiembre 2022. URL: http://journals.openedition.org/jsa/18886 ; DOI: https://doi.org/10.4000/jsa. 18886

All rights reserved 


\title{
Reginaldo Chayax Huex (1939-2020)
}

\author{
Scott Atran, Ximena LoIS \\ y Valentina VAPNARSKY *
}

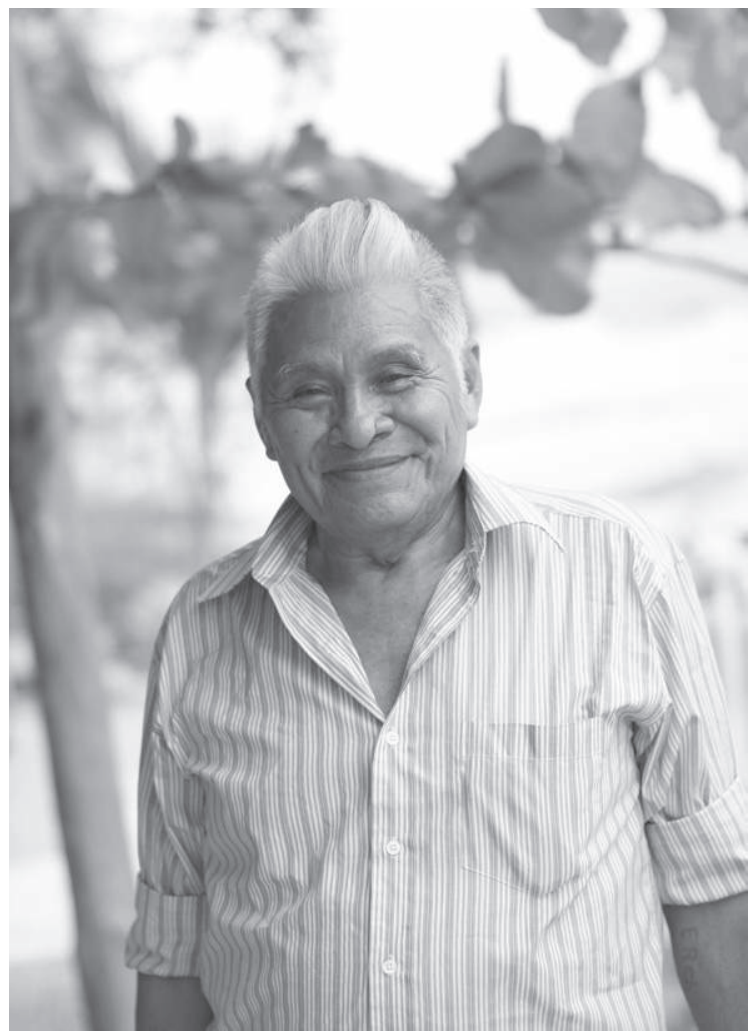

Fig. 1 - Reginaldo Chayax Huex

(foto David Tiago Ribeiro, San José, Petén, Guatemala, 2015)

* S. Atran : Centre for the Study of Social Cohesion, University of Oxford [scott.atran@, pmb.ox.ac.uk] ; X. LoIs : Artis International [xlois@artisinternational.org] ; V.VAPNARSKY : Centre EREA du LESC, Centre nacional de la recherche scientifique (CNRS) [valentina. vapnarsky@cnrs.fr]. 
Don Reginaldo Chayax Huex, líder del pueblo maya itza’ de San José, Petén, Guatemala, y fundador de la Reserva forestal y de la Escuela de lengua BioItzá, falleció a la edad de 81 años el $1^{\circ}$ de octubre de 2020 a consecuencia de la Covid-19. Casado con doña María Espectación Tesucún Chan, tuvo 10 hijos, 28 nietos y 17 bisnietos. Al dolor de su partida se agrega el fallecimiento de su hijo Orlando Chayax Tesucún dos semanas después, así como el de tantos otros mayas víctimas de la pandemia y del fuerte aumento de cánceres en los últimos años debido a la contaminación ecológica del norte de Guatemala ${ }^{1}$. Con la muerte de don Reginaldo también fallece uno de los últimos hablantes del maya itza'. De la generación de quienes hablaron fluidamente la lengua solo queda doña Petrona Vitsil Tesucún, quien, a sus 93 años, acaba de perder en cuatro meses a tres hijos por la pandemia y a una hija por cáncer.

Los itzáes, pueblo del bosque de las tierras bajas, fueron los últimos mayas en ser conquistados por los españoles. A pesar de las duras persecuciones de los gobiernos hasta fines del siglo xx para erradicar su lengua y explotar comercialmente su bosque, los itzáes continuaron comunicándose clandestinamente en lo que las autoridades llamaban "palabras feas" y conservando el legado que les fue dado por los espíritus del bosque, "que existieron incluso antes de Dios". Proteger ese legado era, como le dijera a don Reginaldo su abuela, doña Justa Mex, su más sagrado honor. Don Reginaldo pasó la mayor parte de su vida observando el bosque, desde muy niño en sus andanzas a la milpa y de caza con su padre y como chiclero cuando joven. La otra parte de su vida la pasó cuidándolo. En un encuentro entre itzaés y lacandones en la selva de Najá, Chun K'in Viejo, un centenario líder espiritual de los mayas lacandones del sureste de México dijo una vez sobre don Reginaldo: "en conocimiento del bosque, yo soy su alumno".

A lo largo de su vida, don Reginaldo también fue una referencia de sabiduría y de pericia para los itzáes de su comunidad, así como para los migrantes, esencialmente ladinos y mayas q'eqchíes, que década tras década se instalaron en la región, y recurrían a él en búsqueda de consejos sobre prácticas agrícolas y silvestres. Con su tío, don Domingo Chayax Suntecún, eran unos narradores inagotables de las costumbres de los animales, de las propiedades físicas de las plantas y de los secretos de su óol "corazón y ánimo", del ecosistema silvestre. Del mismo modo, sus descripciones dejaron profundamente admirados a todos los zoólogos y botánicos que las consultaron.

Ciertamente, don Reginaldo fue el maestro de generaciones de investigadores. Fue la primera fuente de inspiración del proyecto "Petén-Itza: Historia Natural de los Mayas de las tierras bajas de Guatemala" (ministère de la Recherche y

1. Liza Grandia, "Toxic Tropics: purity and danger in everywhere in everyday life", Journal of Ecological Anthropology, 21 (1), 2019, p. 1-10 [en línea: https://scholarcommons.usf. edu/jea/vol21/iss1/1, consultado el 01/12/2020]. 
Centre national de la recherche scientifique de Francia [CNRS], University of Michigan y Northwestern University). Esto dio inicio a una larga e intensa serie de proyectos en etnociencia, antropología y psicología cognitiva, psicología intercultural, zoología y botánica, conducidos por instituciones como el CNRS, la Universidad de Michigan, la Northwestern University, la Augustana University y el Artis International. En este marco, don Reginaldo fue un colaborador ineludible, que hoy en día ocuparía naturalmente el lugar de co-autor en muchas de las publicaciones, tales como Plantas de los maya itza' del Petén (Atran, Lois y Ucan 2004). Además de ser una fuente inagotable de conocimientos y de sabiduría, cumplió un papel esencial por su poder de propuesta y de persuasión, por su capacidad inventiva y movilizadora, sin mencionar su gran humor.

A la par de su colaboración en proyectos de investigación, Reginaldo Chayax Huex participó activamente en los esfuerzos de revitalización del idioma itza' que realizó un grupo de mayores, dentro de los últimos hablantes, a partir de la década de 1990, en una época en la que el desprecio y la baja estima hacia esa lengua eran predominantes. Como lo recordaba don Reginaldo en el documental Naachtun (Begoin 2016): "en 1991 nuestro objetivo era rescatar el idioma itza' y que se enseñe en la escuela, pero entonces nos dimos cuenta que también teníamos que rescatar nuestra cultura, no solo nuestra lengua. Y la cultura nuestra está en el bosque, no en la comunidad". Fue en 1991 que creó la Bio-Itzá ${ }^{2}$ con la ayuda de la Universidad de Michigan y con la cooperación de la Agencia de los Estados Unidos para el Desarrollo Internacional (USAID, por sus siglas en inglés). La meta era proveer a los itzáes una estructura que les permitiera desarrollar proyectos a largo plazo para una silvicultura autosostenible, recuperando el conocimiento de la flora y fauna del bosque y su manejo sostenible (Chayax Huex et al. 1999). En este esfuerzo, don Reginaldo defendió por más de treinta años el conocimiento del lazo milenario entre las técnicas mayas de subsistencia y la ecología forestal como legado a las futuras generaciones. En sus propias palabras:

Tan kib 'eetik tulakal umuk'il kisa 'altik yok'la b'aylo' kimejen ch 'ib'alo'ej patal ucha'antiko' b'a'ax tan kimentik...

Estamos dando toda nuestra fuerza para salvarlo, para que así nuestras pequeñas descendencias puedan ver lo que hacemos... (en su texto "Tulakal umuk'il kisa'altik k'aax 'Salvando el bosque con toda nuestra fuerza”', Lois y Vapnarsky 2010, p. 119)

Yankikänäntik a 'k'aaxe, a'k'aax yanukänäntiko'on, men to'one kiwet'ok b'alum, uyumil k'aax, kiwet'ok mo', uyutzilil maaya, kiwet'ok tziminche', ub 'a 'alche' yan tulakal ub'äk'el b'a'alche'oo', kiwet'ok chäkälte', uche'il kich'em, kiwet'ok oox, kijanal, kiwet'ok ub'utz'il uyitz pom, upixan kinukuch taatoo'. 
Much'a'ano'on, tankimentik kiwotoch tuxet'el k'aax, ulu'umil Itza-Maya, tujaal noj ja' Itza', tu'ux käjlajij Kanek', utz'ok unojochil maaya.

Tenemos que cuidar la selva y la selva tiene que cuidarnos, porque somos compañeros del jaguar, dueño de la selva, compañeros de la guacamaya, adorno de los mayas, compañeros del danto, animal de las siete carnes, compañeros de la caoba, árbol de nuestras canoas, compañeros del ramón, nuestra comida, compañeros del humo de la resina del copal, el espíritu de nuestros grandes padres.

Reunidos, estamos haciendo nuestra vivienda en un pedazo de selva, tierra de los Maya-Itzáes, en la orilla de la Gran Agua Itza', donde vivió Kanek, el último de los reyes mayas. (Chayax y Whitacre 1997, p. 1457)

Transmitió este empeño a sus hijos, en particular a su hijo menor Aderito Chayax Tesucún, con quien trabajó mano a mano en la última década y que es hoy responsable de la Bio-Itzá, así como a Orlando Chayax Tesucún. En 1998, se le otorgó a la Bio-Itzá la propiedad en usufructo de un área de bosque de 36 kilómetros cuadrados, la Reserva Comunitaria Indígena Bio-Itzá, que fue la primera reserva comunitaria indígena en establecerse en Guatemala. La BioItzá ha tenido en todos estos años fructuosas cooperaciones con organizaciones nacionales, como Pro-Petén, Conap, Universidad de San Carlos de Guatemala, e internacionales tales como Conservation International, World Wildlife, The Peregrine Fund.

Inspirando con el ejemplo, "hagan lo que yo hago" más que "hagan lo que yo digo", don Reginaldo siempre estaba dispuesto a escuchar lo que pensaban los demás sin desdén ni valoración precipitada, mostrando su confianza en la habilidad de las personas, aprendiendo y enseñando al mismo tiempo. De una fuerza física extraordinaria, sus manos podían limpiar kilómetros de brecha en el bosque en un solo día y aliviar el dolor de huesos y músculos de tantos que venían buscando sus cuidados. Fue amado y admirado más allá de su propio pueblo y respetado por todos los que lo conocieron. Fue un Nojoch Winik, una Gran Persona, que siempre estará en nuestros pensamientos.

\section{Referencias citadas}

Atran Scott, Ximena Lois y Edilberto UCAN Ek

2004 Plants of the Petén Itza' Maya. Plantas de los maya itza' del Petén, Museum of Anthropology, University of Michigan Press (Memoirs, 38), Ann Arbor.

BEGOIN Stéphane

2016 Naachtun, La cité maya oubliée, documental 90 mn., ZED, Arte France, Films à Cinq.

Chayax Huex Reginaldo y David Whitacre

1997 "A plea from the last of the Maya-Itzá", Conservation Biology, 11 (6), p. $1457-1458$. 
Chayax Huex Reginaldo, Feliciano Tzul Coldi, Carlos Gomez Caal y Steven P. Gretzinger 1999 "La reserva Bio-Itzá, historia de un esfuerzo conservacionista indígena de la comunidad maya itzá de San José, Petén, Guatemala" , in Richard B. Primack (ed.), La selva maya. Conservación y desarrollo, Siglo XXI, Madrid, p. 363-373.

LoIs Ximena, y Valentina VAPNARSKY

2010 Itza'. Memorias mayas, fotografías Charles Fréger, introducción Scott Atran, Universidad Nacional Autónoma de México (UNAM), Centro Peninsular en Humanidades y Ciencias Sociales, Mérida/México.

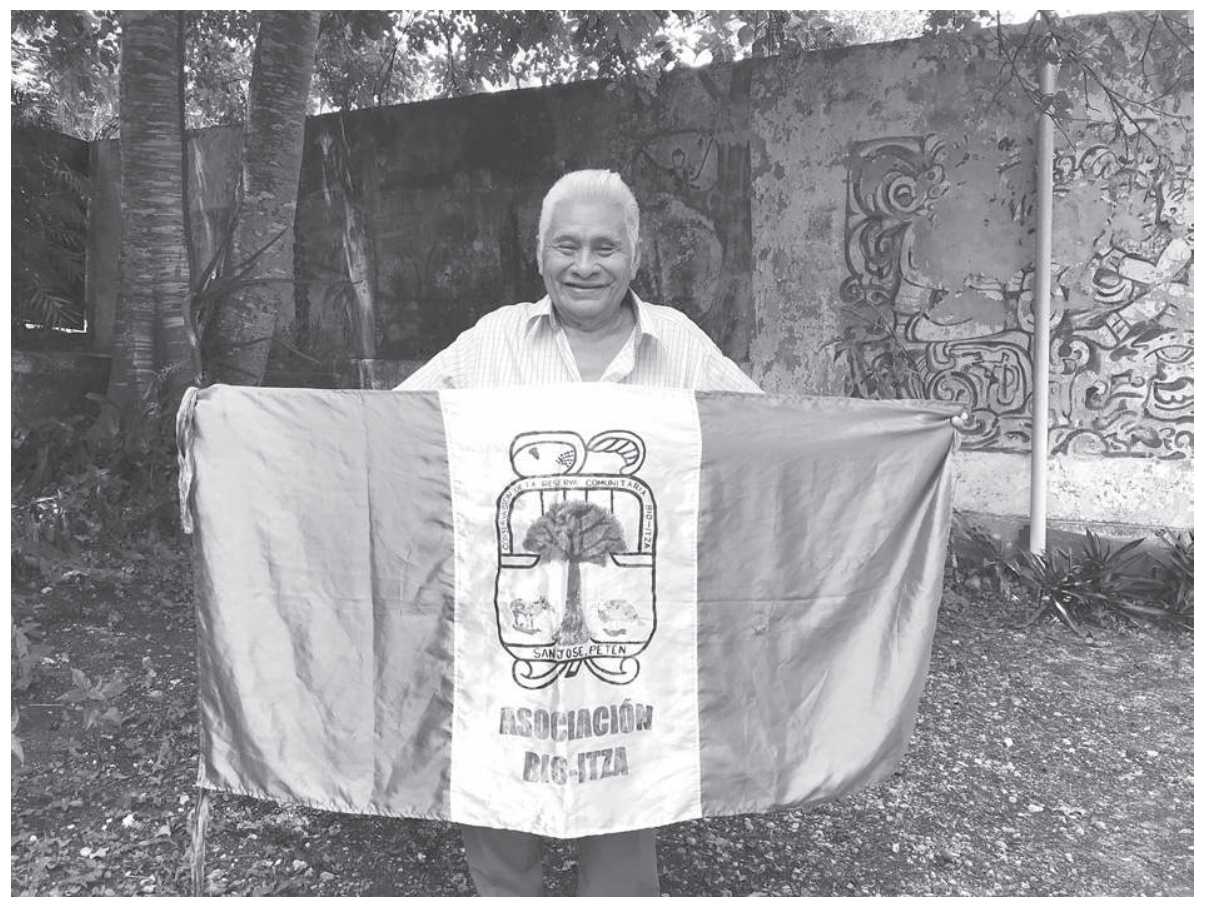

Fig. 2 - Reginaldo Chayax Huex (foto Valentina Vapnarsky, Bio-Itzá, San José, Petén, Guatemala, 2018) 
